\title{
Signal Model and Receiver Algorithms for a Transmit-Reference Ultra-Wideband Communication System
}

\author{
Quang Hieu Dang, António Trindade, Alle-Jan van der Veen, Fellow, IEEE, and Geert Leus, Senior Member, IEEE
}

\begin{abstract}
A communication system based on transmit-reference (TR) ultra-wideband (UWB) is studied and further developed. Introduced by Hoctor and Tomlinson, the aim of the TR-UWB transceiver is to provide a straightforward impulse radio system, feasible to implement with current technology, and to achieve either high data rate transmissions at short distances or low data rate transmissions in typical office or industrial environments. The main contribution in this paper is the derivation of a signal processing model that takes into account the effects of the radio propagation channel, in particular, for the case where the two pulses in a doublet are closely spaced. Several receivers based on the code-division multiple-access-like properties of the proposed model are derived, and the performance of the algorithms is tested in a simulation.
\end{abstract}

Index Terms-Impulse radio, receiver algorithm, signal processing, transmit-reference (TR), ultra-wideband (UWB).

\section{INTRODUCTION}

$\mathbf{T}$ RANSMIT-REFERENCE (TR) systems were devised already in the 1960s as a method for communicating over unknown or random channels [3]. It is known that, in general, the problem of single-user optimal detection leads to the use of a matched filter, i.e., a convolution by the transmitted waveform including the effects of the channel. This waveform is not known and would need to be estimated. The idea of a TR system is that by transmitting a reference signal over the same channel as the message, it can be used in the convolution, so that channel state information is not needed to estimate the information. It is recognized that TR systems may be an inefficient means of transmitting information in a band-limited system [4], with a 3-dB loss in signal-to-noise ratio (SNR) when compared with locally generated reference systems. Nevertheless, the processing constraints of receivers in very high data rate transmissions make this tradeoff worthwhile, as it allows simpler synchronization and channel estimation, especially when compared with RAKE receivers.

Stimulated by the Federal Communications Commission (FCC) notice of proposed rule making, ultra-wideband (UWB) communication technology is now receiving tremendous in-

Manuscript received February 28, 2005; revised November 3, 2005. This paper was supported in part by the Dutch Ministry of Economic Affairs/Ministry of Education Freeband-Impulse Project AIRLINK, and in part by NWO-STW under the VICI program (DTC.5893). This paper was presented in part at the IEEE Conference on Ultra-Wideband Systems and Technologies, Reston, VA, October 2003 and in part at the IEEE International Conference on Ultra-Wideband, Zurich, Switzerland, September 2005.

The authors are with the Department of Electrical Engineering, Delft University of Technology, $2628 \mathrm{CD}$ Delft, The Netherlands (e-mail: allejan@cas.et.tudelft.nl).

Digital Object Identifier 10.1109/JSAC.2005.863828 terest (see [5] for an overview and further references). The first TR-UWB system that can be considered practical was proposed by Hoctor and Tomlinson [6], [7]. Pulses are transmitted in pairs (referred to as "doublets"), where the first is fixed and considered a "carrier" and the second is modulated by the data. The delay between the pulses can be varied, which serves as a user code. The receiver correlates the received data with several shifts of it using a bank of correlation lags, integrates, samples, and digitally combines the outputs of the bank. The features of this system are reduced requirements for synchronization at the receiver, sampling and digital processing at a feasible rate, and the use of straightforward nonadaptive analog components.

In their paper, Hoctor and Tomlinson propose a simple receiver structure based on a simplified matched filter. However, they did not take the effect of the propagation channel into account. The delay spread of measured channels can be up to about $200 \mathrm{~ns}$ [8], much longer than the time interval between two pulses in a doublet, which, by design, is in the order of a nanosecond. This introduces additional correlations which have a detrimental effect on the detection. In this paper, we extract these correlation coefficients from experimental data, and compare this to the analytic results presented by Witrisal et al. in [9].

A comparison of UWB suboptimal receivers in realistic channels is done in [10]. In contrast to most descriptions of UWB systems that are in continuous time, a discrete-time model for pulse position modulation impulse radio multiple access is developed in [11] (cf. [12] for additional references). In this paper, we extend both approaches with a proposal for an accurate signal processing data model for the TR UWB system, specifically for the case where the two pulses in a doublet are closely spaced. The model takes the propagation channel into account, and maps it into a specific set of "effective channel coefficients," in fact correlation coefficients. These can be estimated from the received data of a single symbol. With a more accurate data model, it is straightforward to design improved receivers, from the matched filter to a blind iterative receiver. Although readily extendable, our current model is limited in the sense that, here, we consider only a single-user system. Interference of other UWB signals or concurrent narrowband systems (e.g., CDMA, Bluetooth, WiFi, GPS) is not yet taken into account. In [13], a generalization to TR-UWB is proposed where a frame can contain more than two pulses and the receiver utilizes a bank of correlators; our model is usually not applicable to this system unless the transmitted signal is designed such that the received signal is linear in the transmitted symbols.

This paper expands on the work in [1] and [2], and proposes a compact data model for a TR UWB system (Section II), including the effect of dispersive channels (Section III). Based 


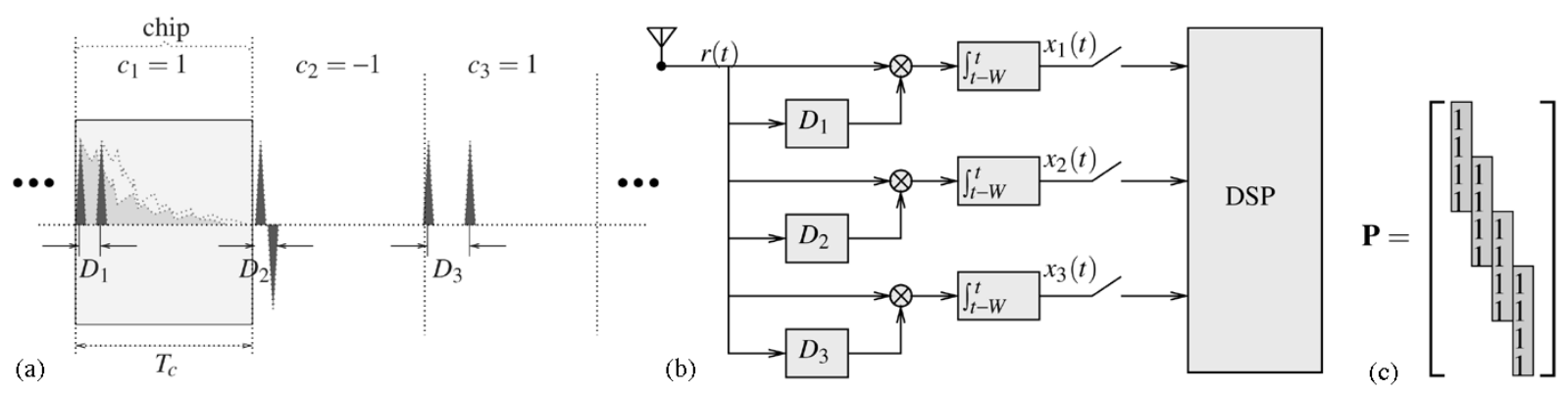

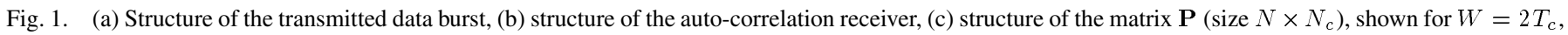
$P=2$, and $N_{c}=4$.

on this, we derive several receiver algorithms (Section IV). The proposed algorithms are blind or semiblind: the channel parameters (in this case correlations) are estimated along with the data. Section V shows the simulated performance of the algorithms.

\section{TRANSMIT-REFERENCE (TR) DATA MODEL}

We consider a single-user delay-hopped transmit reference system as originally proposed in [6], and develop its signal processing model (as in [1]). In a delay-hopped TR system, the transmitted signal consist of a sequence of $N_{c}$ chips, each of duration $T_{c}$. To simplify the presentation, we first consider the data model for a single chip.

\section{A. Single Chip}

As depicted in Fig. 1(a), for each chip a pair (doublet) of narrow pulses $g(t)$ is transmitted, spaced by a time interval of duration $D_{i}$, selected from a collection $\left\{D_{1}, \ldots, D_{M}\right\}$, where we assume $D_{1}<D_{2}<\cdots<D_{M}$. The first pulse is fixed, whereas the second pulse is modulated by the chip value $c \in$ $\{+1,-1\}$. For the $j$ th chip, transmitted at time instant $t=j T_{c}$, the chip value is $c_{j}$ and the selected delay is $i=i(j)$ (following a user-dependent chip sequence and index function), and can be written as

$$
c_{j}(t)=g\left(t-j T_{c}\right)+c_{j} g\left(t-j T_{c}-D_{i}\right) .
$$

Let $h_{p}(t)$ be the radio propagation channel, and define the convolution between a monopulse and the physical channel as $h(t)=g(t) * h_{p}(t)$, which we assume to have duration $T_{h}$. Ignoring the additive noise (see [14] for this extension) the received signal for the transmitted chip (1) can then be expressed as

$$
r_{j}(t)=h\left(t-j T_{c}\right)+c_{j} h\left(t-j T_{c}-D_{i}\right) .
$$

At the receiver, it is passed through a bank of $M$ correlators, each correlating the signal with a delayed version of itself at lags $D_{m}, m=1, \ldots, M$. Subsequently, the outputs of the correlators are integrated over a sliding window of duration $W \geq T_{c}$, as in Fig. 1(b). The output of the $m$ th correlator and integrator branch for the received signal (2) can then be written as

$$
\begin{aligned}
x_{m, j}(t)= & \int_{t-W}^{t} r_{j}(\tau) r_{j}\left(\tau-D_{m}\right) d \tau \\
= & \int_{t-j T_{c}-W}^{t-j T_{c}} r_{j}\left(\tau+j T_{c}\right) r_{j}\left(\tau+j T_{c}-D_{m}\right) d \tau \\
= & \kappa\left(t-j T_{c}, D_{m}\right)+\kappa\left(t-j T_{c}-D_{i}, D_{m}\right) \\
& +c_{j}\left[\kappa\left(t-j T_{c}-D_{i}, D_{m}-D_{i}\right)\right. \\
& \left.\quad+\kappa\left(t-j T_{c}, D_{m}+D_{i}\right)\right]
\end{aligned}
$$

where

$$
\kappa(t, \Delta)=\int_{t-W}^{t} h(\tau) h(\tau-\Delta) d \tau .
$$

Assuming that $W$ is larger than the channel duration $T_{h}$, it is straightforward to derive that

$$
\kappa(t, \Delta)= \begin{cases}0, & t \leq 0 \\ \rho(\Delta), & T_{h}<t<W \\ 0, & t>W+T_{h}\end{cases}
$$

where

$$
\rho(\Delta)=\int_{0}^{\infty} h(\tau) h(\tau-\Delta) d \tau
$$

and with channel-dependent values in the unspecified intervals. Assuming furthermore that $W$ is not just larger but much larger than the channel duration $T_{h}$, it is thus seen that $\kappa(t, \Delta)$ is well approximated by a "brick function" $p(t)$ which is independent of $\Delta$

$$
p(t)= \begin{cases}0, & t \leq 0, t \geq W \\ 1, & 0<t<W\end{cases}
$$

times a scaling $\rho(\Delta)$, so that

$$
\kappa(t, \Delta) \approx p(t) \rho(\Delta) .
$$

Under this approximation, and assuming that $W$ is also much larger than the maximal delay $D_{M}$, which implies $\kappa\left(t-j T_{c}-\right.$ $\left.D_{i}, \Delta\right) \approx \kappa\left(t-j T_{c}, \Delta\right)$, the output of the $m$ th correlator and integrator branch (3) can be rewritten as

$$
\begin{aligned}
x_{m, j}(t)= & p\left(t-j T_{c}\right) \\
& \times\left\{2 \rho\left(D_{m}\right)+c_{j}\left[\rho\left(D_{m}-D_{i}\right)+\rho\left(D_{m}+D_{i}\right)\right]\right\} \\
= & p\left(t-j T_{c}\right)\left(c_{j} \alpha_{m, i}+\beta_{m}\right)
\end{aligned}
$$

where

$$
\begin{aligned}
\alpha_{m, i} & =\rho\left(D_{m}-D_{i}\right)+\rho\left(D_{m}+D_{i}\right) \\
\beta_{m} & =2 \rho\left(D_{m}\right) .
\end{aligned}
$$

Note that $\alpha_{m, i}=\alpha_{i, m}$, while $\beta_{m}$ only depends on $D_{m}$. We may interpret $\alpha_{m i}$ as a channel gain, whereas $\beta_{m}$ is an offset. These unknown parameters replace the usual channel coefficients. Similarly, the "brick function" $p(t)$ plays the role of "pulse shape function" in the model for $x_{m, j}(t)$.

If $\alpha_{m, i}=\alpha \delta_{m, i}$ where $\alpha=\int h^{2}(t) d t$ is the channel energy and $\delta$ is the Kronecker delta function, and if $\beta_{m}=0$, then we obtain the data model considered by Hoctor and Tomlinson in [6] and [7]. In this case, we simply have $x_{m, j}(t)=p(t-$ $\left.j T_{c}\right) c_{j} \alpha \delta_{m, i}$, with a nonzero output only if the transmit delay matches the receiver delay. For channels with a short duration 
$T_{h}$ (compact support for the correlation function), this model is a good approximation. For channels with a longer impulse response (in the order of the maximal delay $D_{M}$, or larger), this model may be too simple. The statistics of these parameters will be further studied in Section III.

\section{B. Multiple Chips-Matrix Formulation}

Let us now consider transmitting a symbol $s \in\{+1,-1\}$. This is done by transmitting $N_{c}$ consecutive chips $\mathbf{c}=$ $\left[c_{0}, \ldots, c_{N_{c}-1}\right]^{T}$ multiplied by the symbol $s$. Each chip is transmitted using one of the delays $D_{1}, \ldots, D_{M}$ and is received using a bank of $M$ correlators at delays $D_{1}, \ldots, D_{M}$. Based on (9), and assuming $T_{c}$ is larger than the channel duration $T_{h}$ plus twice the maximal delay $D_{M}\left(T_{c}>T_{h}+2 D_{M}\right)$, in order to avoid overlap between consecutive chips after correlation, we can write the output of the $m$ th correlator and integrator branch for the symbol $s$ as

$$
x_{m}(t)=\sum_{i=1}^{M} \sum_{j=0}^{N_{c}-1} p\left(t-j T_{c}\right)\left(\alpha_{m, i} J_{i, j} c_{j} s+\beta_{m} J_{i, j}\right)
$$

where

$$
J_{i, j}=\left\{\begin{array}{ll}
1, & \text { if chip } j \text { is transmitted at delay } D_{i} \\
0, & \text { elsewhere }
\end{array} .\right.
$$

Assume that the outputs of the integrators are sampled at $P$ times the chip rate, where $P$ is the oversampling rate (typically $P=2$ ). The sampled data at the instances $t=n T_{c} / P+\varepsilon$ is then given by

$$
\begin{aligned}
x_{m, n} & =x_{m}\left(\frac{n T_{c}}{P}+\varepsilon\right) \\
& =\sum_{i=1}^{M} \sum_{j=0}^{N_{c}-1} p_{n, j}\left(\alpha_{m, i} J_{i, j} c_{j} s+\beta_{m} J_{i, j}\right)
\end{aligned}
$$

where $p_{n, j}=p\left(n T_{c} / P+\varepsilon-j T_{c}\right)$. Here, $n$ is an integer and $\varepsilon$ is a fractional offset, $\varepsilon \in\left[0, T_{c} / P\right)$.

To obtain a matrix model for the symbol $s$, we will collect $N=N_{c} P$ temporal samples at the output of the $m$ th correlator and integrator branch into the vector $\mathbf{x}_{m}=\left[x_{m, 0}, \ldots, x_{m, N-1}\right]^{T}$. Let us further define the $M \times 1$ channel vector $\mathbf{a}_{m}$ as $\left[\mathbf{a}_{m}\right]_{i}=\alpha_{m, i}$ and the $M \times M$ channel matrix $\mathbf{A}$ as $[\mathbf{A}]_{m, i}=\alpha_{m, i}$ (note that $\mathbf{A}=\mathbf{A}^{T}$ since $\left.\alpha_{m, i}=\alpha_{i, m}\right)$. In addition, we define the $M \times 1$ channel vector $\mathbf{b}$ as $[\mathbf{b}]_{m}=\beta_{m}$. To describe the delay code, we also define the $M \times N_{c}$ selector matrix $\mathbf{J}$ as $[\mathbf{J}]_{i, j+1}=J_{i, j}$. It has for each column only one nonzero entry, corresponding to the transmitted delay index at that chip. Therefore, $\mathbf{J}^{T} \mathbf{1}_{M}=\mathbf{1}_{N_{c}}$. Finally, define the $N \times N_{c}$ sampled pulse matrix $\mathbf{P}$ as $[\mathbf{P}]_{n+1, j+1}=p_{n, j}$, the structure of which is shown in Fig. 1(c).

The above definitions allow us to express $\mathbf{x}_{m}=$ $\left[x_{m, 0}, \ldots, x_{m, N-1}\right]^{T}$ as

$$
\begin{aligned}
\mathbf{x}_{m}= & \sum_{i=1}^{M} \sum_{j=0}^{N_{c}-1} \mathbf{p}_{j}\left(\alpha_{m, i} J_{i, j} c_{j} s+\beta_{m} J_{i, j}\right) \\
= & {\left[\mathbf{p}_{0} c_{0}, \ldots, \mathbf{p}_{N_{c}-1} c_{N_{c}-1}\right] \mathbf{J}^{T} \mathbf{a}_{m} s } \\
& +\left[\mathbf{p}_{0}, \ldots, \mathbf{p}_{N_{c}-1}\right] \mathbf{J}^{T} \mathbf{1}_{M} \beta_{m} \\
= & \mathbf{P} \operatorname{diag}(\mathbf{c}) \mathbf{J}^{T} \mathbf{a}_{m} s+\mathbf{P} \mathbf{1}_{N_{c}} \beta_{m}
\end{aligned}
$$

where $\mathbf{p}_{j}$ is the $(j+1)$ st column of $\mathbf{P}$. Collecting all vectors $\mathbf{x}_{m}$ into a matrix $\mathbf{X}=\left[\mathbf{x}_{1}, \ldots, \mathbf{x}_{M}\right]$ gives

$$
\mathbf{X}=\mathbf{P} \operatorname{diag}(\mathbf{c}) \mathbf{J}^{T} \mathbf{A}^{T} s+\mathbf{P} \mathbf{1}_{N_{c}} \mathbf{b}^{T} .
$$

Finally, if we transmit multiple symbols $\mathbf{s}=\left[s_{0}, \ldots, s_{N_{s}-1}\right]^{T}$, and assume there is no overlap between consecutive symbols (this can be obtained by inserting a guard interval of $\left\lceil W / T_{c}\right\rceil$ zeros (blank chips) in between every two symbols), we have for the $k$ th symbol

$$
\begin{aligned}
\mathbf{X}_{k} & =\mathbf{P} \operatorname{diag}(\mathbf{c}) \mathbf{J}^{T} \mathbf{A}^{T} s_{k}+\mathbf{P} \mathbf{1}_{N_{c}} \mathbf{b}^{T} \\
& =\mathbf{P}\left[\operatorname{diag}(\mathbf{c}) \mathbf{J}^{T}, \mathbf{1}_{N_{c}}\right]\left[\mathbf{A} s_{k}, \mathbf{b}\right]^{T} .
\end{aligned}
$$

For simplicity, we assumed here that periodic codes are used. In this receiver model, $\mathbf{X}_{k}$ is measured, $\mathbf{c}$ is known (user code), $\mathbf{J}$ is known (delay code), and $\mathbf{P}$ is known and data independent (this assumes synchronization; without synchronization an unknown number of zero rows are stacked on top but this can be estimated and resolved, see [15]). $\mathbf{A}$ and $\mathbf{b}$ are unknown (channel correlation coefficients), and $s_{k}$ is the data symbol to be detected.

\section{Remarks and Extensions}

For the simple data model considered by Hoctor and Tomlinson [6], [7], i.e., assuming no correlations for unmatched delays, we obtain $\mathbf{A}=\alpha \mathbf{I}$ and $\mathbf{b}=0$. For channels with an impulse response longer than $D_{M}$, this may not be a valid assumption. This is studied in more detail in Section III.

The advantage of the receiver structure is that it is data independent and nonadaptive. Even synchronization is not needed in the analog domain; this can be done in the DSP based on the received data model [15]. With $P=2$ times oversampling of the integrator output, there is no loss of information.

The typical duration of the integration window is $W=T_{c}$. If the receiver uses an integrate-and-dump operation (which resets the integrator after sampling), then without oversampling $(P=1)$ the model remains the same. Technologically, such integrators have the advantage that the integration length is easily modified (related to an external clock).

In some descriptions of TR systems, multiple doublets per chip are considered. This may be useful for increased range/low data rate applications. It is a special case of the above model, with duplicate values for the chips and delays. Alternatively, it can be modeled using a triangular "tent shape" for $p(t)$ [1].

At the receiver, it is essential that a low-pass filter be used prior to the correlation, to limit the noise. Finally, in practical systems, it is advisable to randomize the polarity of the first (reference) pulse as well, which will reduce spectral lines. In the noise-free case, this has no influence on the model after the correlator

\section{Channel Model and Statistics}

In Section II-A, it was shown that, by correlation and integration, the effect of the propagation channel on the data model will reduce to the parameters $\alpha_{m i}$ and $\beta_{m}$, which depend on the effective channel autocorrelation function $\rho(\Delta)$, see (10). In this section, we will study the expected value and variance of these parameters under certain statistical assumptions of the physical channel. 
TABLE I

VAlue of $\Phi$ For Some Pulses With Normalized ENERGY

\begin{tabular}{lcc}
\hline Pulse type & Width & $\Phi$ \\
\hline Rectangular & $T$ & $2 / 3 T$ \\
Manchester & $T$ & $1 / 3 T$ \\
Gaussian pulse & $\approx 4 \tau_{m}$ & $\tau_{m} \sqrt{\pi}$ \\
2nd order Gaussian monocycle & $\approx 3 \tau_{m}$ & $\approx 0.94 \tau_{m}$ \\
\hline
\end{tabular}

We consider a multipath channel model, where the physical channel impulse response is modeled as a sum of discrete delta pulses

$$
h_{p}(t)=\sum_{i=0}^{\infty} a_{i} \delta\left(t-\tau_{i}\right)
$$

where $a_{i}$ are ray amplitudes, and $\tau_{i}$ are their corresponding arrival times. Generally, these parameters are considered as random variables with different statistical assumptions depending on the specific channel model. A typical channel model for UWB is assumed to be time-invariant and to have uncorrelated ray amplitudes $a_{i}$, where ray amplitudes will be negligibly small for large $\tau_{i}$.

\section{A. Statistics of $\rho(\Delta)$ for Uncorrelated Channel Taps With Exponential Decay}

The case of an exponentially decaying power delay profile in relation to a TR-UWB system was studied in detail in [9], and some of their resulting expressions are summarized below. The channel autocorrelation function defined in (6) depends on both the physical channel response and the transmitted UWB pulse $g(t)$. With the physical channel model in (15), the effective channel response is

$$
h(t):=g(t) * h_{p}(t)=\sum_{i=0}^{\infty} a_{i} g\left(t-\tau_{i}\right) .
$$

The expected value of $\rho(\Delta)$ is

$$
E[\rho(\Delta)]=\int_{0}^{\infty} E[h(\tau) h(\tau-\Delta)] d \tau .
$$

If we assume uncorrelated channel taps, i.e., $E\left[a_{i} a_{j}\right]=0$ for $i \neq j$, then [9]

$$
E[\rho(\Delta)]=P_{0} \phi_{g}(\Delta)
$$

where $P_{0}$ is the total received power in $h_{p}(t)$, whereas

$$
\phi_{g}(\Delta):=\int_{0}^{\infty} g(\tau) g(\tau-\Delta) d \tau
$$

is the autocorrelation of the transmitted UWB pulse. Note that $\phi_{g}(\Delta)=0$ for $\Delta \geq T_{g}$, where $T_{g}$ is the pulse duration. For typical pulses, $T_{g}$ will be short, and only evaluation of $\rho(\Delta)$ at a discrete set of lags $\Delta$ is needed, equal to the sums and differences of the delays used in the transceiver. Assuming the minimum difference in lags is larger than $T_{g}$, effectively $E[\rho(\Delta)]$ is nonzero only for $\Delta=0$.

In [9], also explicit expressions for the variance $\operatorname{var}(\rho(\Delta))$ are derived, under the assumptions that the channel has an exponentially decaying power delay profile with parameter $\gamma$ plus a line-of-sight (LOS) component with power ratio (Ricean factor)
$K$. Furthermore, the arrival density of rays is assumed to be $\lambda$ rays/s. The variance of $\rho(\Delta)$ for $\Delta>T_{g}$ is then shown to be

$$
\operatorname{var}[\rho(\Delta)]=\gamma P_{0}^{2} \Phi \frac{2 K+1}{2(K+1)^{2}} e^{-\gamma \Delta}, \quad\left(\Delta>T_{g}\right)
$$

where $\Phi:=\int_{-\infty}^{\infty} \phi_{g}^{2}(\kappa) d \kappa$ relates to the bandwidth of the UWB pulse. For $\Delta \stackrel{-\infty}{=}$

$$
\operatorname{var}[\rho(0)] \approx \frac{\gamma P_{0}^{2}}{(K+1)^{2}}\left[\Phi(2 K+1)+\frac{\Psi}{\lambda}\right]
$$

where

$$
\Psi:=\int_{-T_{g}}^{T_{g}} \phi_{g^{2}}(\varepsilon) d \varepsilon=\int_{-T_{g}}^{T_{g}} \int_{0}^{T_{g}} g^{2}(t) g^{2}(t-\varepsilon) d t d \varepsilon .
$$

$\Phi$ and $\Psi$ depend only on the transmitted pulse; for a unit-energy pulse $\Psi=1$. For such a pulse, some typical values of $\Phi$ are shown in Table I. In the table, $\tau_{m}$ is the parameter of the Gaussian monocycle (or second derivative of a Gaussian pulse), i.e., $g(t)=\left[1-4 \pi\left(t / \tau_{m}\right)^{2}\right] e^{-2 \pi\left(t / \tau_{m}\right)^{2}}$.

As an example, consider a Gaussian monocycle with $\tau_{m}=$ $0.2 \mathrm{~ns}$, and a multipath channel with parameters $P_{0}=1$ (normalized channel power), $K=0$ (nonline-of-sight channel), $\tau_{\text {rms }}=1 / \gamma=15 \mathrm{~ns}, \lambda=5 \mathrm{~ns}^{-1}$. In this case, $E(\rho(0))=1$, whereas $E(\rho(\Delta))=0,(\Delta \neq 0)$. Similarly, $\sqrt{\operatorname{var}(\rho(0))} \approx$ 0.16 , whereas $\sqrt{\operatorname{var}(\rho(\Delta))} \approx 0.07,(\Delta \neq 0)$. Thus, according to this model, $\rho(\Delta)$ is significant only for $\Delta=0$, which gives credibility to the model assumptions considered by Hoctor and Tomlinson [6], [7].

\section{B. Statistics of $\alpha_{m i}$ and $\beta_{m}$ for Uncorrelated Taps With Exponential Decay}

Based on the statistics of $\rho(\Delta)$, it is straightforward to derive the expectations and variances of $\alpha_{m i}$ and $\beta_{m}$. Substituting the mean values of $\rho(\Delta)$ into (10), we have

$$
\begin{aligned}
E\left[\alpha_{m i}\right] & = \begin{cases}0, & \text { for } m \neq i \\
P_{0} \phi_{g}(0), & \text { for } m=i\end{cases} \\
E\left[\beta_{m}\right] & =0 .
\end{aligned}
$$

Similarly, the variances become

$$
\begin{aligned}
\sigma^{2}\left[\alpha_{m i}\right] & = \begin{cases}\frac{\gamma \ell P_{0}^{2}}{2} \Phi\left(e^{-\gamma\left(\left|D_{i}-D_{m}\right|\right)}+e^{-\gamma\left(D_{m}+D_{i}\right)}\right), & \text { for } m \neq i \\
\gamma P_{0}^{2} \ell\left[\Phi\left(1+\frac{1}{2} e^{-2 \gamma D_{m}}\right)+\frac{\Psi}{2 \lambda(K+1)}\right], & \text { for } m=i\end{cases} \\
\sigma^{2}\left[\beta_{m}\right] & =2 \gamma \ell P_{0}^{2} \Phi e^{-\gamma D_{m}} \\
\text { where } \ell & =(2 K+1) /(K+1)^{2} .
\end{aligned}
$$

\section{Measured Channel Correlation Coefficients}

To inspect the autocorrelation function $\rho(\tau)$ for more realistic channels, we consider a realization of the CM-1 physical channel model based on the IEEE 802.15.3a standard [16] (LOS, 0-4 m). To obtain the effective channel response $h(t)$, the physical channel needs to be convolved with the transmitted pulse shape $g(t)$ and twice with a wideband antenna response, to include the effect of the transmit and receive antennas. Although in the UWB literature the antenna is often modeled as a simple differentiator, we will use here an actual measured response of a biconical antenna, as shown in Fig. 2(a). This includes the response of a bandpass filter used to limit interference from wireless local area network (WLAN) equipment and 
(a)

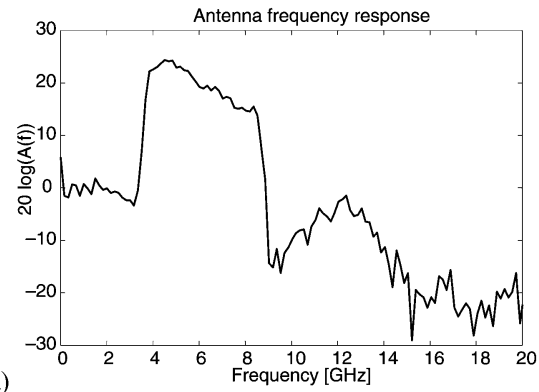

Fig. 2. (a) Measurement antenna transfer function, including bandpass filter. (b) Autocorrelation function for an IEEE CM 1 channel (LOS), including pulse shaping and transmit/receive antenna/bandpass filter response. (c) Idem for measured channel "API 3" (LOS).

out-of-band noise. ${ }^{1}$ The corresponding impulse response has a duration of about $1.5 \mathrm{~ns}$. For the transmitted pulse, we take a Gaussian pulse with parameter $\tau_{m}=0.2 \mathrm{~ns}$.

The resulting function $\rho(\tau)$ for a single realization of the CM-1 model is shown in Fig. 2(b). We also show $\rho(\tau)$ for a channel impulse response measured in a $40-\mathrm{m}$ wide and $15-\mathrm{m}$ high industrial hall ("API" at TU Delft) containing several machines for the process industry [see Fig. 2(c)]. This particular measurement is a LOS scenario over a distance of $9 \mathrm{~m}$ using a very narrow pulse, and has offline been convolved with a Gaussian pulse $\left(\tau_{m}=0.2 \mathrm{~ns}\right)$. We only show the segment of interest, i.e., small values of $\tau$ up to a few times the pulse width, since it is hard to implement integrated delay lines with wideband delays much longer than this.

The figures show that $\rho(0)$ is dominant and typically only 3-5 times larger than the other values of $\rho(\tau)$ for $\tau>1 \mathrm{~ns}$, compared with a factor 15 for the theoretical channel model in Section III-A. A major cause for this is the spreading of the pulse introduced by the antenna, thus violating the assumption in the theoretical model that $T_{g}<\Delta$.

It is also seen that the correlation peak at 0 is very narrow (less than $100 \mathrm{ps).} \mathrm{Typical} \mathrm{delay} \mathrm{lines} \mathrm{that} \mathrm{can} \mathrm{be} \mathrm{integrated} \mathrm{on} \mathrm{a} \mathrm{chip}$ (e.g., RC filters) have tolerances that are higher than this. If this peak is somehow missed, e.g., due to mismatch in the transmit and receive delays, then $\rho(\tau)$ for any (small) value of $\tau$ may be significant. Consequently, all values of $\alpha_{m i}, \beta_{m}$ are significant in this case; one cannot assume that $\mathbf{A}$ is diagonally dominant and that $\mathbf{b}$ is zero.

\section{RECEIVER ALGORITHMS}

Based on the data model derived in Section II, we can now develop a number of detection algorithms. Augmented with noise terms, the data model (14) is

$$
\mathbf{X}_{k}=\mathbf{P} \operatorname{diag}(\mathbf{c}) \mathbf{J}^{T} \mathbf{A} s_{k}+\mathbf{P} \mathbf{1}_{N_{c}} \mathbf{b}^{T}+\mathbf{N}_{k}
$$

where $\mathbf{N}_{k}$ collects all noise terms after correlation/integration (this includes signal-noise and noise-noise terms). We will assume that the data symbols $s_{k}$ are drawn from a binary phase-shift keying (BPSK) constellation. The problem now is, given the received signal $\mathbf{X}_{k}$, estimate $s_{k}$ along with the unknown channel matrix $\mathbf{A}$ and channel vector $\mathbf{b}$. Depending on the knowledge we have on the statistics of $\mathbf{N}_{k}$ (this knowledge

\footnotetext{
${ }^{1}$ We are grateful to Z. Irahhauten, G. Janssen, and A. Yarovoy for implementing and conducting these experiments.
}

could be obtained by training; see also [14]), we can whiten it or not. The algorithms listed below will for simplicity assume that $\mathbf{N}_{k}$ is white.

\section{A. Simplified Matched-Filter Receiver}

A simple receiver can be derived if we assume that the channel does not have temporal correlations $\left(\alpha_{m, i}=\alpha \delta_{m, i}\right)$. In that case, the channel matrix and offset vector will be $\mathbf{A}=\alpha \mathbf{I}, \mathbf{b}=\mathbf{0}$, where $\alpha>0$ is the only unknown constant (the channel power). The resulting simplified data model is

$$
\mathbf{X}_{k}=\mathbf{P} \operatorname{diag}(\mathbf{c}) \mathbf{J}^{T} \alpha s_{k}+\mathbf{N}_{k}
$$

which leads to a corresponding matched-filter receiver

$$
\alpha \hat{s}_{k}=\operatorname{tr}\left[\mathbf{J} \operatorname{diag}(\mathbf{c}) \mathbf{P}^{T} \mathbf{X}_{k}\right]
$$

where $\operatorname{tr}(\cdot)$ is the trace operator. Since $\alpha$ is always positive, it does not change the detected symbol for our assumed BPSK constellation and, thus, it does not need to be estimated. ${ }^{2}$

\section{B. Blind Multiple Symbol Receiver}

If $\mathbf{A}$ and $\mathbf{b}$ are unknown, they can be estimated along with the data $\mathbf{s}=\left[s_{0}, \ldots, s_{N_{s}-1}\right]^{T}$ in a blind scheme as follows. Write the model as

$$
\begin{aligned}
{\left[\mathbf{X}_{0}, \ldots, \mathbf{X}_{N_{s}-1}\right]=} & \mathbf{P}\left[\operatorname{diag}(\mathbf{c}) \mathbf{J}^{T}, \mathbf{1}\right] \\
& \times\left[\begin{array}{ccc}
\mathbf{A}^{T} s_{0} & \ldots & \mathbf{A}^{T} s_{N_{s}-1} \\
\mathbf{b}^{T} & \ldots & \mathbf{b}^{T}
\end{array}\right]+\mathbf{N}
\end{aligned}
$$

where $\mathbf{N}=\left[\mathbf{N}_{0}, \ldots, \mathbf{N}_{N_{s}-1}\right]$. Since $\mathbf{Q}:=\mathbf{P}\left[\operatorname{diag}(\mathbf{c}) \mathbf{J}^{T}, \mathbf{1}\right]$ is completely known, we can remove its effect by multiplying both sides with the left pseudoinverse of $\mathbf{Q}$ (assuming it is tall, i.e., $N \geq M+1$ )

$$
\begin{aligned}
{\left[\mathbf{Y}_{0}, \ldots, \mathbf{Y}_{N_{s}-1}\right] } & :=\mathbf{Q}^{\dagger}\left[\mathbf{X}_{0}, \ldots, \mathbf{X}_{N_{s}-1}\right] \\
& =\left[\begin{array}{ccc}
\mathbf{A}^{T} s_{0} & \ldots & \mathbf{A}^{T} s_{N_{s}-1} \\
\mathbf{b}^{T} & \ldots & \mathbf{b}^{T}
\end{array}\right]+\mathbf{Q}^{\dagger} \mathbf{N} .
\end{aligned}
$$

It is then clear that the channel vector $\mathbf{b}$ can be estimated by averaging the last rows of the matrices $\mathbf{Y}_{k}$, i.e., using a Matlab notation

$$
\hat{\mathbf{b}}^{T}=\frac{1}{N_{s}} \sum_{k=0}^{N_{s}-1}\left[\mathbf{Y}_{k}\right]_{M+1,:}
$$

${ }^{2}$ Note that this receiver structure uses only one delay output at a time; thus it does not correspond to a true matched filter, but to a matched filter for a simplified model. 
To estimate $\mathbf{A}$ and $\mathbf{s}$, we vectorize the matrices $\left[\mathbf{Y}_{k}\right]_{1: M,:}$ into vectors $\mathbf{y}_{k}=\operatorname{vec}\left(\left[\mathbf{Y}_{k}\right]_{1: M,:}\right)$ of size $M^{2} \times 1$ by stacking its columns, and define $\mathbf{Y}^{\prime}=\left[\mathbf{y}_{0}, \ldots, \mathbf{y}_{N_{s}-1}\right]$. This matrix has model

$$
\mathbf{Y}^{\prime}=\operatorname{vec}\left(\mathbf{A}^{T}\right) \mathbf{s}^{T}+\mathbf{N}^{\prime}
$$

where $\mathbf{N}^{\prime}$ is similarly defined as $\mathbf{Y}^{\prime}$, but based on $\mathbf{Q}^{\dagger} \mathbf{N}$. Hence, the channel matrix $\mathbf{A}$ and the source symbol vector $\mathbf{s}$ can be estimated up to a scaling by computing a rank-1 decomposition (using the SVD) of $\mathbf{Y}^{\prime}$. For a BPSK constellation, the scaling is easily established.

\section{Iterative Receiver}

In the preceding receiver algorithm, the inversion of $\mathbf{Q}$ may be undesirable (e.g., it may not be a very tall matrix, and a poorly conditioned inverse will enhance and color the noise). Improved performance can be obtained by a two-step iterative receiver which is initialized by the receiver of the preceding section: 1) assume s is known, estimate $\mathbf{A}$ and $\mathbf{b} ; 2$ ) assume $\mathbf{A}$ and $\mathbf{b}$ are known, estimate $\mathbf{s}$. For the first step, we rewrite the data model (18) as

$\left[\begin{array}{c}\mathbf{X}_{0} \\ \vdots \\ \mathbf{X}_{N_{s}-1}\end{array}\right]=\left[\begin{array}{c}\mathbf{P}\left[s_{0} \operatorname{diag}(\mathbf{c}) \mathbf{J}^{T}, \mathbf{1}\right] \\ \vdots \\ \mathbf{P}\left[s_{N_{s}-1} \operatorname{diag}(\mathbf{c}) \mathbf{J}^{T}, \mathbf{1}\right]\end{array}\right]\left[\begin{array}{c}\mathbf{A}^{T} \\ \mathbf{b}^{T}\end{array}\right]+\left[\begin{array}{c}\mathbf{N}_{0} \\ \vdots \\ \mathbf{N}_{N_{s}-1}\end{array}\right]$

from which $\mathbf{A}$ and $\mathbf{b}$ can be estimated using least squares as

$$
\left[\begin{array}{c}
\hat{\mathbf{A}}^{T} \\
\hat{\mathbf{b}}^{T}
\end{array}\right]=\left[\begin{array}{c}
\mathbf{P}\left[s_{0} \operatorname{diag}(\mathbf{c}) \mathbf{J}^{T}, \mathbf{1}\right] \\
\vdots \\
\mathbf{P}\left[s_{N_{s}-1} \operatorname{diag}(\mathbf{c}) \mathbf{J}^{T}, \mathbf{1}\right]
\end{array}\right]^{\dagger}\left[\begin{array}{c}
\mathbf{X}_{0} \\
\vdots \\
\mathbf{X}_{N_{s}-1}
\end{array}\right] .
$$

The matrix which is inverted has size $N N_{s} \times(M+1)$ and should be tall $(N N s \geq M+1)$. For the second step, we partition $\mathbf{Q}$ in (18) as $\mathbf{Q}=\left[\mathbf{Q}^{\prime}, \mathbf{q}\right]$ and obtain

$$
\operatorname{vec}\left(\mathbf{X}_{k}\right)=\operatorname{vec}\left(\mathbf{Q}^{\prime} \mathbf{A}^{T}\right) s_{k}+\mathbf{b} \otimes \mathbf{q}+\operatorname{vec}\left(\mathbf{N}_{k}\right)
$$

where $\otimes$ denotes a Kronecker product. Therefore, a least squares solution for $s_{k}$ is

$$
\hat{s}_{k}=\left[\operatorname{vec}\left(\mathbf{Q}^{\prime} \mathbf{A}^{T}\right)\right]^{\dagger}\left(\operatorname{vec}\left(\mathbf{X}_{k}\right)-\mathbf{b} \otimes \mathbf{q}\right)
$$

which is straightforward to evaluate.

\section{Simulation Results}

We simulate the transmission of $N_{s}=20$ symbols over the UWB channels described in Section III. We consider the IEEE CM-1 (LOS) channel, convolved with a Gaussian pulse and twice with the measured antenna/bandpass filter response; furthermore, we consider the API-3 measured channel convolved with the same Gaussian pulse. We use 100 Monte Carlo runs to obtain the BER versus SNR plots for the various receiver algorithms, while the channel is kept fixed. Here, the SNR is defined as the average received energy in a symbol over the white Gaussian noise power density.

The system uses $M=3$ delay positions, and $N_{c}=5$ chips per symbol. The transmitted Gaussian pulse has duration parameter $\tau_{m}=0.2 \mathrm{~ns}$. The two pulses in a doublet are separated by $D_{m} \in\{0.5,1.0,1.5\} \mathrm{ns}$, and the doublets are separated by $T_{c}=70 \mathrm{~ns}$ to avoid interframe interference. The integration interval is taken as $W=T_{c}$, and no oversampling is used $(P=1)$.

The receiver algorithms which are tested are the simplified matched-filter receiver (Section IV-A), which uses a single (matched) delay per received chip, the blind multiple symbol receiver (Section IV-B), which uses the complete bank of receiver delays for each received chip, and the iterative receiver (Section IV-C), which uses the complete data model and is initialized by either one of the two noniterative receivers.

Fig. 3(a) shows the BER versus the SNR for various algorithms for the IEEE CM-1 channel. The channel matrices in this case are

$$
\mathbf{A}=\left[\begin{array}{ccc}
0.969 & -0.071 & -0.038 \\
-0.071 & 0.993 & -0.092 \\
-0.038 & -0.092 & 0.962
\end{array}\right], \quad \mathbf{b}=\left[\begin{array}{c}
-0.207 \\
-0.061 \\
0.066
\end{array}\right]
$$

Similarly, Fig. 3(c) shows the results for the API-3 measured channel, for which

$$
\mathbf{A}=\left[\begin{array}{ccc}
0.984 & 0.171 & -0.003 \\
0.171 & 1.013 & -0.015 \\
-0.003 & -0.015 & 1.038
\end{array}\right], \quad \mathbf{b}=\left[\begin{array}{c}
0.008 \\
-0.032 \\
0.335
\end{array}\right]
$$

In both cases, the figures show that the simplified matched-filter receiver is more accurate than the blind multisymbol receiver (BMSR). Postprocessing with the iterative algorithm (which uses the full signal model) provides little advantage. Thus, the assumption that $\mathbf{A}=\alpha \mathbf{I}$ and $\mathbf{b}=\mathbf{0}$ is sufficiently accurate. The relatively poor performance of the BMSR is explained from the fact that $\mathbf{Q}$ in this case has size $5 \times 4$, which is not very tall; thus, some noise enhancement will occur. The iterative receiver instead inverts a matrix which grows with the number of samples and, therefore, experiences less noise enhancement in the estimation of $(\mathbf{A}, \mathbf{b})$ in (21). The detection step (22) involves the "inversion" of a vector which is always well conditioned as it only depends on the total amount of energy collected in the correlation bank.

We next consider the case where there is a small timing offset in each receiver delay due to component inaccuracies. For the IEEE CM-1 channel model, we take the offset as small as $0.05 \mathrm{~ns}$, for the measured API channel, we take it perhaps more realistically equal to $0.2 \mathrm{~ns}$. As discussed in Section III, due to this offset the diagonal dominance property of the channel matrix $\mathbf{A}$ is affected. The resulting channel correlation matrix is for the IEEE CM-1 channel

$$
\mathbf{A}=\left[\begin{array}{ccc}
-0.225 & -0.121 & 0.081 \\
0.246 & -0.214 & -0.117 \\
0.043 & 0.197 & -0.241
\end{array}\right], \quad \mathbf{b}=\left[\begin{array}{c}
-0.256 \\
0.069 \\
0.014
\end{array}\right]
$$

and for the measured API-3 channel

$$
\mathbf{A}=\left[\begin{array}{ccc}
0.311 & 0.148 & 0.008 \\
-0.149 & 0.415 & 0.058 \\
0.018 & -0.240 & 0.391
\end{array}\right], \quad \mathbf{b}=\left[\begin{array}{c}
0.115 \\
-0.097 \\
0.182
\end{array}\right] \text {. }
$$

Fig. 3(b) and (d) shows the results. It is seen that, for the CM-1 channel, the simplified matched-filter receiver completely breaks down since it assumes $\mathbf{A}=\alpha \mathbf{I}, \mathbf{b}=\mathbf{0}$ which is not at all accurate, whereas the BMSR, which takes into account all the elements of matrices $\mathbf{A}$ and $\mathbf{b}$, maintains a fair performance. A less strong conclusion holds for the API channel. In both cases, the iterative algorithm gives a significant performance improvement over both noniterative algorithms. As Fig. 2(b) 

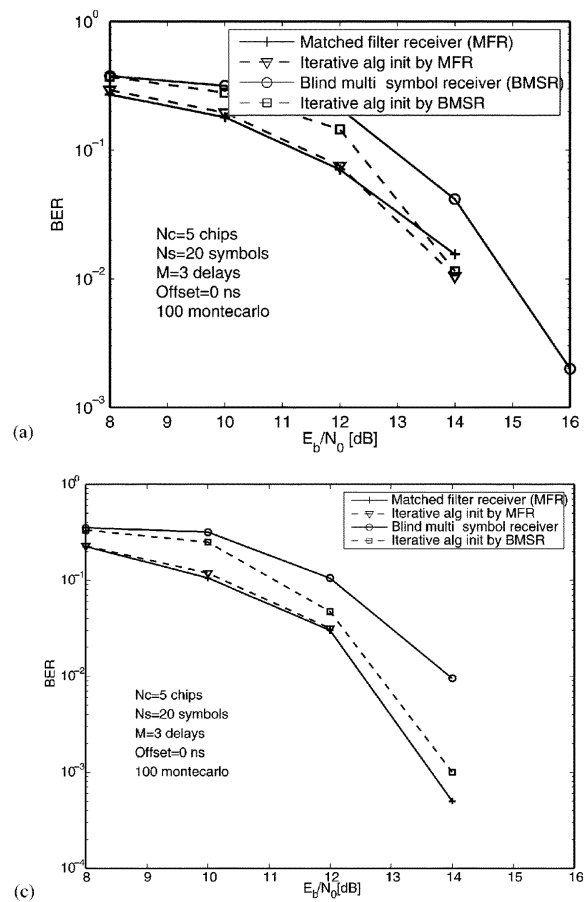
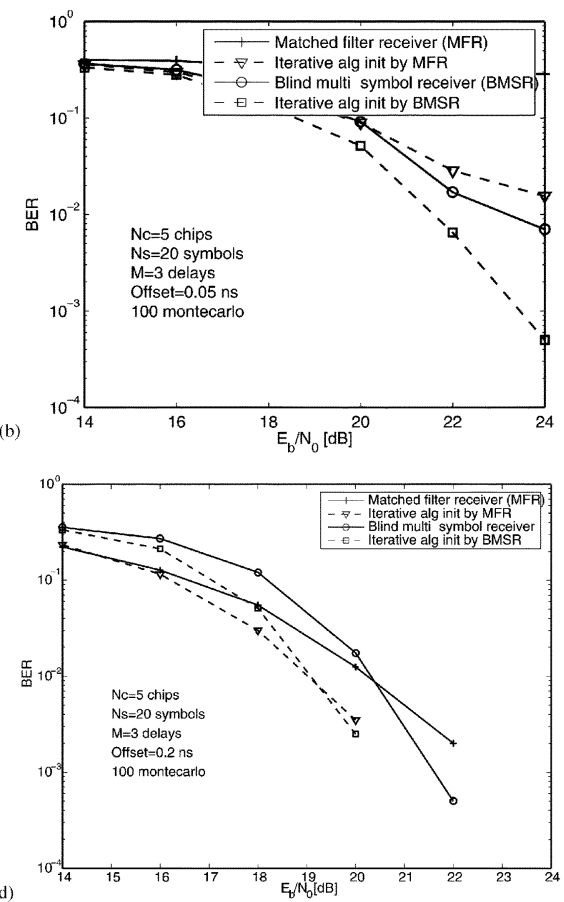

Fig. 3. BER versus SNR for different receiver algorithms. IEEE CM-1 channel (LOS) including antenna/filter response. (a) No delay mismatch. (b) Delay mismatch 0.05 ns. Measured channel (“API 3," LOS). (c) No delay mismatch. (d) Delay mismatch 0.2 ns.

and (c) shows, the values of $(\mathbf{A}, \mathbf{b})$ strongly depend on precisely which delays (values of $\tau$ ) are selected. Only receivers which use the full data model are expected to be resilient to this.

\section{CONCLUSION}

We have proposed an accurate signal processing model for a TR-UWB system, in particular, for the case where both pulses in a doublet are more closely spaced than the length of the impulse response. The model considers the channel correlation coefficients, which can be estimated blindly from a single symbol or multiple symbols, and used in a simplified matched-filter receiver or in a more advanced multiple symbol or iterative receiver. The performance of the iterative receiver is as good and occasionally much better than the matched-filter and the BMSR. Additional work shows that joint timing acquisition and detection based on this model is fairly straightforward [15]. Future work will provide a multiuser analysis, and pay attention to interchip interference.

\section{REFERENCES}

[1] A. Trindade, Q. H. Dang, and A. J. van der Veen, "Signal processing model for a transmit-reference UWB wireless communication system," in Proc. IEEE Conf. Ultra Wideband Syst. Technol., Reston, VA, Oct. 2003, pp. 270-274.

[2] Q. H. Dang, A. Trindade, and A. J. van der Veen, "Considering delay inaccuracies in a transmit-reference UWB communication system," in Proc. IEEE Int. Conf. UWB, Zurich, Switzerland, Sep. 2005, pp. 582-586.

[3] C. K. Rushforth, "Transmitted-reference techniques for random or unknown channels," IEEE Trans. Inf. Theory, vol. IT-10, no. 1, pp. 39-42, Jan. 1964.

[4] R. M. Gagliardi, "A geometrical study of transmitted reference communication systems," IEEE Trans. Commun. Technol., vol. 12, no. 4, pp. 118-123, Dec. 1964

[5] S. Roy, J. Foerster, V. S. Somayazulu, and D. G. Leeper, "Ultrawideband radio design: The promise of high-speed, short-range wireless connectivity," Proc. IEEE, vol. 92, no. 2, pp. 295-311, Feb. 2004.

[6] R. Hoctor and H. Tomlinson, "Delay-hopped transmitted-reference RF communications," in Proc. IEEE Conf. Ultra Wideband Syst. Technol., 2002, pp. 265-270.
[7] N. van Stralen, A. Dentinger, K. Welles, R. Gauss, R. Hoctor, and H. Tomlinson, "Delay hopped transmitted reference experimental results," in Proc. IEEE Conf. Ultra Wideband Syst. Technol., 2002, pp. 93-98.

[8] D. Cassioli, M. Win, and A. Molisch, "The ultra-wide bandwidth indoor channel: From statistical model to simulations," IEEE J. Sel. Areas Commun., vol. 20, no. 6, pp. 1247-1257, Aug. 2002.

[9] K. Witrisal, M. Pausini, and A. Trindade, "Multiuser interference and inter-frame interference in UWB transmitted reference systems," in Proc. IEEE Conf. Ultra Wideband Syst. Technol., Kyoto, Japan, May 2004, pp. 96-100.

[10] J. Choi and W. Stark, "Performance of ultra-wideband communications with suboptimal receivers in multipath channels," IEEE J. Sel. Areas Commun., vol. 20, no. 9, pp. 1754-1766, Dec. 2002.

[11] L. Yang and G. Giannakis, "Multistage block-spreading for impulse radio multiple access through ISI channels," IEEE J. Sel. Areas Commun., vol. 20, no. 9, pp. 1767-1777, Dec. 2002.

[12] - "Ultra-wideband communications-An idea whose time has come," IEEE Signal Process. Mag., no. 6, pp. 26-54, Nov. 2004.

[13] H. Zhang and D. L. Goeckel, "Generalized transmit-reference UWB systems," in Proc. IEEE Conf. Ultra Wideband Syst. Technol., Reston, VA, Oct. 2003, pp. 147-151.

[14] Q. H. Dang, A. J. van der Veen, and A. Trindade, "Statistical analysis of a transmit-reference UWB wireless communication system," in Proc. IEEE ICASSP, Philadelphia, PA, Mar. 2005, pp. iii/317-iii/317.

[15] R. Djapic, G. Leus, and A. J. van der Veen, "Blind synchronization in asynchronous UWB networks based on the transmit-reference scheme," in Proc. Asilomar Conf. Signals, Syst. Comput., Nov. 2004 pp. $1506-1510$.

[16] A. F. Molisch, J. R. Foerster, and M. Pendergrass, "Channel models for ultrawideband personal area networks," IEEE Pers. Commun. Mag., vol. 10, no. 6, pp. 14-21, Dec. 2003.

Quang Hieu Dang, photograph and biography not available at the time of publication.

António Trindade, photograph and biography not available at the time of publication.

Alle-Jan van der Veen (S'88-M'88-SM'02-F'05), photograph and biography not available at the time of publication.

Geert Leus (SM'05), photograph and biography not available at the time of publication. 